\title{
Efficacy of light based detection systems for early detection of oral cancer and oral potentially malignant disorders: Systematic review
}

\author{
Ravleen Nagi 1, Yashoda-Bhoomi Reddy-Kantharaj ${ }^{2}$, Nagaraju Rakesh ${ }^{3}$, Sujatha Janardhan-Reddy ${ }^{4}$, \\ Shashikant Sahu ${ }^{5}$
}

\author{
${ }^{1}$ Senior Lecturer. Department of Oral Medicine and Radiology, New Horizon Dental College and Research Institute, Sakri, \\ Bilaspur, Chattisgarh \\ ${ }^{2}$ Senior Professor. Department of Oral Medicine and Radiology, Faculty of Dental Sciences, MS Ramaiah University of Ap- \\ plied Sciences, MSRIT Post, Mathikere, Bangalore, Karnataka, India \\ ${ }^{3}$ Reader. Department of Oral Medicine and Radiology, Faculty of Dental Sciences, MS Ramaiah University of Applied Sci- \\ ences, MSRIT Post, Mathikere, Bangalore, Karnataka, India \\ ${ }^{4}$ Professor and Head. Department of Oral Medicine and Radiology, Faculty of Dental Sciences, MS Ramaiah University of Ap- \\ plied Sciences, MSRIT Post, Mathikere, Bangalore, Karnataka, India \\ ${ }^{5}$ Consultant. Plastic Surgeon, Burn and Trauma Centre, Bilaspur, Chattisgarh
}

Correspondence:

Department of oral Medicine and Radiology

New Horizon Dental

College and Research Institute

Sakri, Bilaspur, India

ravleennagi@yahoo.in

Received: $15 / 10 / 2015$

Accepted: 23/12/2015

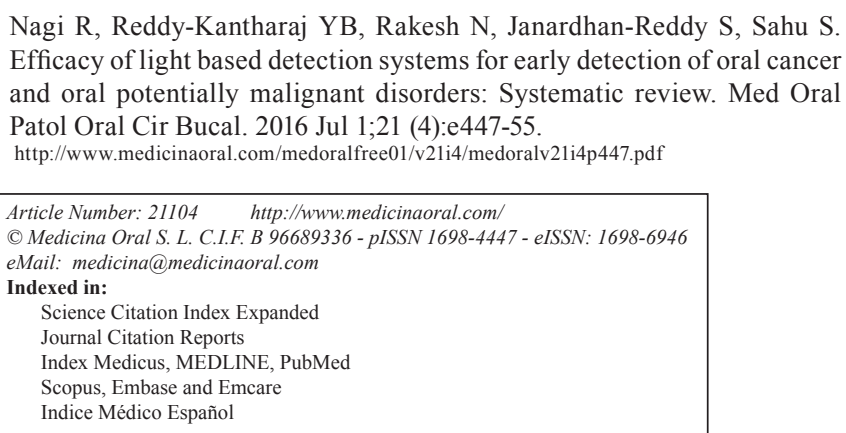

Nagi R, Reddy-Kantharaj YB, Rakesh N, Janardhan-Reddy S, Sahu S. Efficacy of light based detection systems for early detection of oral cancer Patol Oral Cir Bucal. 2016 Jul 1;21 (4):e447-55. http://www.medicinaoral.com/medoralfree 01/v21i4/medoralv21i4p447.pdf

\begin{abstract}
Background: Earlier detection of oral squamous cell carcinoma (OSCC) and oral potentially malignant disorders (OPMD) is essential for dental professionals to improve patient survival rates. The aim of this systematic review is to to evaluate the effectiveness of devices that utilise the principles of chemiluminescence and tissue autofluorescence as adjuncts in the detection of OSCC and OPMD.

Material and Methods: The electronic retrieval systems and databases searched for relevant articles were PubMed [MEDLINE] and Science direct. The search was for limited articles published in English or with an English abstract and articles published during the period from January 2005 to April 2014. Clinical trials utilized ViziLite, Microlux TM/DL and Visual Enhanced Light scope (VELscope) for early detection of OPMD and OSCC.

Results: Twenty primary studies published satisfied our criteria for selection - 10 utilised chemiluminescence and 10 tissue autofluorescence. Senstivity of Vizilite for detecting OSCC nad OPMD ranged from $77.1 \%$ to $100 \%$ and specificity was low that ranged from $0 \%$ to $27.8 \%$. Most have shown that chemiluminescence increases the brightness and margins of oral mucosal white lesions and thus assist in identification of mucosal lesions not considered under Conventional visual examination. However, it preferentially detects leukoplakia and may fail to spot red patches. Clinical trials demonstrated that sensitivity of VELscope in detecting malignancy and OPMD ranged from $22 \%$ to $100 \%$ and specificity ranged from $16 \%$ to $100 \%$. Most studies concluded that VELscope can help the experienced clinician to find oral precursor malignant lesions. But it couldnot differentiate between dysplasia and benign inflammatory conditions.
\end{abstract}


Conclusions: Both devices are simple, non-invasive test of the oral mucosa but are suited for clinicians with sufficient experience and training. More clinical trials in future should be conducted to establish optical imaging as an efficacious adjunct tool in early diagnosis of OSCC and OPMD.

Key words: Oral cancer, early diagnosis, potentially malignant disorders, chemiluminescence, tissue autofluorescence, VELscope, ViziLite plus.

\section{Introduction}

Oral malignancies are one of the most common cancers around the world and ranks sixth to eighth among cancers in various studies. These cancers are major economic and clinical burden for the health care around the world (1). In India, oral cancer represents a major health problem accounting for upto $40 \%$ of all cancers, and is most common cancer in males and third most common cancer in females. It often arises from Oral potential malignant disorders (OPMDs) such as erythroplakia, leukoplakia and oral Lichen planus (2). Leukoplakia is the most common OPMD and its worldwide prevalence is approximately $2.6 \%$ (3).

Risk factors for oral cancer are well established and include tobacco and alcohol use (4). Despite the established risk factors and advances in treatment, the 5-year survival for oral squamous cell carcinoma (OSCC) associated with tobacco and alcohol use has remained consistently poor for the last forty years (5). Prognosis is further complicated by the high rate of second primary tumours in these patients, which is thought to be the result of 'field cancerisation' in the upper aerodigestive tract (6).

Early detection of neoplastic changes in the oral cavity is the best method to improve patient survival rates (7). The current method of oral cancer diagnosis, visual examination of the oral cavity, relies heavily on clinical expertise in recognizing early neoplastic changes. However, discerning premalignant and early malignant lesions from common benign inflammatory conditions by visual examination is difficult, even for experienced practitioners (8). Many techniques to date have been reviewed so far e.g. vital staining procedure (Toulidine Blue and Lugols iodine), Brush Biopsy (Oral CDx Brush),micronuclei anlaysis, DNA ploidy but have certain limitations (2). Light-based techniques, including chemiluminescence and autofluorescent imaging, work on the assumption that neoplastic and pre-neoplastic tissues that have undergone abnormal metabolic or structural changes have different absorbance and reflectance properties when exposed to specific wavelengths of light. In the last decade, light-based technology has been adapted and marketed for use in the oral cavity (chemiluminescence: ViziLite, ViziLite Plus, MicroLuxTM/DL; autofluorescence: VELscope (Visual Enhanced Light scope) (9). The objective of this systematic review is to evaluate the literature investigating the effectiveness of chemiluminescence and autofluorescent imaging devices as aids in the detection of OSCC and OPMDs and encouraging dental professionals to use these light based detection devices in clinical practice.

\section{Material and Methods}

A systematic review of the scientific literature was done in preparation of manuscript. The electronic retrieval systems and databases searched for relevant articles were PUBMED [MEDLINE] and SCIENCE DIRECT. Database of indexed journals were searched for keywords such as Oral cancer, early diagnosis, potentially malignant disorders, chemiluminescence; tissue autofluorescence, VELscope; ViziLite Plus. The inclusion criteria were the use of light based techniques for early diagnosis of OSCC or OPMD, publications reporting primary studies and publications written in English. The exclusion criteria were case reports, reviews and studies in other languages.

\section{Results}

For the use of chemiluminescence aids (ViziLite, ViziLite plus and MicroluxTM DL) in the detection of OPMD and OSCC ten studies satisfied our inclusion and exclusion criteria. These studies were conducted in clinics of countries such as Malaysia (10), Australia $(11,12)$, USA (13), India (14-16) and UK (17). Most studies used ViziLite to detect OPMD and OSCC but one study used Microlux TM/DL (12). Table 1 and 1 continue, illustrates the clinical trials conducted in literature establishing the role of chemiluminescence in detection of OPMD and OSCC.

Most studies were cross sectional studies and several parameters were considered for correct evaluation. The sensitivity of a test, is the proportion of people who test positive for a specific disease among a group of people who have the disease. Specificity is the proportion of people who test negative for a specific disease among a group of people who do not have the disease. False positive is an erroneously positive test or screening result. False nega- 
Table 1. Summarizes the clinical trials to evaluate the efficacy of chemiluminescence in detection of oral cancer and oral potentially malignant disorders.

\begin{tabular}{|c|c|c|c|c|c|c|}
\hline $\begin{array}{l}\text { Author } \\
\text { (year) }\end{array}$ & Study design & $\begin{array}{l}\text { Sample, Selection } \\
\text { criteria }\end{array}$ & Intervention & $\begin{array}{l}\text { Outcome: } \\
\text { senstivity }\end{array}$ & $\begin{array}{l}\text { Outcome: } \\
\text { specificity }\end{array}$ & $\begin{array}{l}\text { Summary of findings, } \\
\text { Conclusion }\end{array}$ \\
\hline $\begin{array}{l}\text { Ram S et al. } \\
2005 \text { ( 10) }\end{array}$ & $\begin{array}{l}\text { Cross- } \\
\text { sectional } \\
\text { study }\end{array}$ & $\begin{array}{l}6 \text { patients (OSCC } \\
14,26 \text { OPMD, } 6 \\
\text { benign) } \\
\text { Selection criteria } \\
\text { Oral mucosal } \\
\text { white lesion } \\
\end{array}$ & $\begin{array}{l}\text { Chemiluminescence } \\
\text { as an diagnostic aid } \\
\text { for detection of oral } \\
\text { cancer and } \\
\text { potentially } \\
\text { malignant epithelial } \\
\text { lesions }\end{array}$ & $100 \%$ & $14.2 \%$ & $\begin{array}{l}\text { Conclusion: } \\
\text { chemiluminescence is a more } \\
\text { reliable diagnostic tool than } \\
\text { tolonium chloride in the } \\
\text { detection of oral cancer and } \\
\text { PMELs, and for follow-up of } \\
\text { patients treated for the same. }\end{array}$ \\
\hline $\begin{array}{l}\text { Farah and Mc } \\
\text { Cullough. } 2007 \\
\text { (11) }\end{array}$ & $\begin{array}{l}\text { Cross- } \\
\text { sectional } \\
\text { study }\end{array}$ & $\begin{array}{l}55 \text { patients(1 } \\
\text { OSCC, } 9 \text { dysplasias, } \\
45 \text { benign lesions) } \\
\\
\text { Selection criteria: } \\
\text { Oral mucosal } \\
\text { white lesion }\end{array}$ & $\begin{array}{l}\text { Efficacy of acetic } \\
\text { acid wash and } \\
\text { chemiluminescent } \\
\text { illumination } \\
\text { (ViziLite } \\
\text { Trademark) in the } \\
\text { visualization of oral } \\
\text { mucosal white } \\
\text { lesions }\end{array}$ & $\begin{array}{l}\text { OSCC:100 } \\
\% \\
\text { Dysplasa:1 } \\
00 \%\end{array}$ & $0 \%$ & $\begin{array}{l}\text { OSCC } \\
\text { All cases were viziLite } \\
\text { positive. No false negatives. } \\
\text { Benign } \\
\text { All benign lesion were } \\
\text { ViziLite positive.This } \\
\text { represented } 45 \text { false positive } \\
\text { screens } \\
\text { Conclusion } \\
\text { The device doesnot help in } \\
\text { identification of malignant } \\
\text { and potentially malignant } \\
\text { lesions }\end{array}$ \\
\hline $\begin{array}{l}\text { Epstein et al. } \\
2008(13)\end{array}$ & $\begin{array}{l}\text { Cross } \\
\text { sectional } \\
\text { study }\end{array}$ & $\begin{array}{l}84 \text { patients }(9 \\
\text { OSCC, } 4 \text { CIS, } 41 \\
\text { dysplasia, } 43 \\
\text { benign) } \\
\text { Selection criteria: } \\
\text { Oral mucosal } \\
\text { white lesion } \\
\text { Previous history of } \\
\text { OSCC or high risk } \\
\text { patients }\end{array}$ & $\begin{array}{l}\text { Analysis of oral } \\
\text { lesion biopsies } \\
\text { identified and } \\
\text { evaluated by visual } \\
\text { examination, } \\
\text { chemiluminescence } \\
\text { and toulidine blue }\end{array}$ & $\begin{array}{l}\text { Not } \\
\text { reported }\end{array}$ & Not reported & $\begin{array}{l}\text { OSCC/OPMD } \\
\text { All were ViziLite positive } \\
\text { Benign } \\
\text { All lesions without dysplasia } \\
\text { were ViziLite positive } \\
\text { representing } 43 \text { percent false } \\
\text { positive screens. } \\
\text { TBlue } \\
\text { Identified } 1 / 3 \text { benign } \\
\text { lesions, } 58 \% \text { mild to moderate } \\
\text { lesions and } 100 \% \text { of Severe } \\
\text { pathologies } \\
\text { Conclusion } \\
\text { TBlue reduced the number of } \\
\text { false positive screens }\end{array}$ \\
\hline $\begin{array}{l}\text { McIntosh et al. } \\
2009 \text { (12) }\end{array}$ & $\begin{array}{l}\text { Cross } \\
\text { sectional } \\
\text { study }\end{array}$ & $\begin{array}{l}50 \text { patients( } 2 \\
\text { OSCC, } 7 \\
\text { dysplasia,41 } \\
\text { benign) } \\
\text { Selection criteria: } \\
\text { Oral mucosal } \\
\text { white lesion }\end{array}$ & $\begin{array}{l}\text { Assessment of } \\
\text { diffused light } \\
\text { illumination and } \\
\text { acetic acid rinse } \\
\text { (Microlux/DL }{ }^{\mathrm{TM}} \text { ) in } \\
\text { the visualization of } \\
\text { oral mucosal lesions }\end{array}$ & $\begin{array}{l}\text { Dysplasia/ } \\
\text { OSCC: } \\
77.8 \%\end{array}$ & $70.7 \%$ & $\begin{array}{l}\text { OSCC/OPMD } \\
\text { Microlux /DL showed no } \\
\text { positive result in } 2 \text { dysplastic } \\
\text { lesions. } \\
\text { Benign } \\
12 \text { benign lesions elicited } \\
\text { ViziLite positive responses } \\
\text { Conclusion: } \\
\text { Unable to discriminate } \\
\text { malignancy,OPMD, benign } \\
\text { keratosis, inflammatory } \\
\text { conditions }\end{array}$ \\
\hline $\begin{array}{l}\text { Mehrotra et al. } \\
2010(14)\end{array}$ & $\begin{array}{l}\text { Cross } \\
\text { sectional } \\
\text { study }\end{array}$ & $\begin{array}{l}102 \text { patients ( } 1 \\
\text { OSCC, } 3 \\
\text { dysplasia, } 98 \\
\text { benign) } \\
\text { Selection criteria: } \\
\text { Oral mucosal } \\
\text { white lesion }\end{array}$ & $\begin{array}{l}\text { Evaluation of } \\
\text { chemiluminescence } \\
\text { and auto } \\
\text { fluorescence in the } \\
\text { detection of } \\
\text { clinically innocuous } \\
\text { precancerous and } \\
\text { cancerous oral } \\
\text { lesions }\end{array}$ & $\begin{array}{l}\text { OSCC : } 0 \% \\
\text { Dysplasia:0 } \\
\%\end{array}$ & $75.5 \%$ & $\begin{array}{l}\text { OSCC/OPMD } \\
\text { The device failed to detect all } \\
3 \text { cases of dysplasia and } 1 \\
\text { case of OSCC representing } 4 \\
\text { false negative screens } \\
\text { Benign: } \\
24 \text { of } 74 \text { benign lesions elicited } \\
\text { ViziLite positive response } \\
\text { Conclusion: } \\
\text { ViziLite plus system offers no } \\
\text { benefit in detecting OSCC and } \\
\text { OPMD }\end{array}$ \\
\hline
\end{tabular}


Table 1 Continue. Summarizes the clinical trials to evaluate the efficacy of chemiluminescence in detection of oral cancer and oral potentially malignant disorders.

\begin{tabular}{|c|c|c|c|c|c|c|}
\hline $\begin{array}{l}\text { Awan et al. } \\
2011 \text { (17) }\end{array}$ & $\begin{array}{l}\text { Cross } \\
\text { sectional } \\
\text { study }\end{array}$ & $\begin{array}{l}126 \text { patients } \\
\text { OPMD/benign (61 } \\
\text { leukoplakia,9eryth } \\
\text { roplakia, 32 lichen } \\
\text { planus/lichenoid } \\
\text { reaction,9 chronic } \\
\text { hyperplastic } \\
\text { candidiasis,2 oral } \\
\text { submucous } \\
\text { fibrosis) } \\
44 \text { dysplasia, } 56 \\
\text { benign } \\
\text { Selection criteria: } \\
\text { Patients with } \\
\text { white, red , mixed } \\
\text { red and white } \\
\text { Patches. }\end{array}$ & $\begin{array}{l}\text { Utility of } \\
\text { chemiluminescence } \\
\text { (ViziLite) in the } \\
\text { detection of orally } \\
\text { potential malignant } \\
\text { disorders and } \\
\text { benign keratoses }\end{array}$ & $\begin{array}{l}\text { Dysplasia } \\
77.3 \% \\
\text { Leuko/eryh } \\
\text { troplakia: } \\
77.1 \%\end{array}$ & $\begin{array}{l}\text { Dysplasia: } \\
27.8 \% \\
\text { Leuko/erythr } \\
\text { oplakia: } \\
26.8 \%\end{array}$ & $\begin{array}{l}\text { OPMD/DYSPLASIA } \\
80.3 \% \text { of leukoplakia lesions } \\
\text { elicited ViziLite positive } \\
\text { result.Fifty percent of } \\
\text { erythroplakia lesions elicited } \\
\text { vizilite positive result } \\
\text { Benign } \\
52 \text { benign positive ViziLite } \\
\text { response } \\
\text { Conclusion: } \\
\text { The device has low specificity } \\
\text { for dysplasia and is poor at } \\
\text { detecting some red lesiuons }\end{array}$ \\
\hline $\begin{array}{l}\text { Ujaoney et al. } \\
2012 \text { (15) }\end{array}$ & $\begin{array}{l}\text { Cross } \\
\text { sectional } \\
\text { study }\end{array}$ & $\begin{array}{l}50 \text { patients } \\
\text { (OPMD/benign) } \\
\text { Selection criteria: } \\
\text { Patients with at } \\
\text { least one } \\
\text { precancerous } \\
\text { lesion }\end{array}$ & $\begin{array}{l}\text { Evaluation of } \\
\text { chemiluminescsnce, } \\
\text { toulididine blue and } \\
\text { histopathology for } \\
\text { detection of high } \\
\text { risk oral } \\
\text { precancerous } \\
\text { lesions }\end{array}$ & $\begin{array}{l}\text { Vizilite } \\
: 100 \% \\
\text { TBlue:59\% }\end{array}$ & $\begin{array}{l}\text { Vizilite: } 1 \% \\
\text { Tblue:79\% }\end{array}$ & $\begin{array}{l}\text { Conclusion: } \\
\text { Toluidine blue retention test } \\
\text { may be better suited than } \\
\text { chemiluminescence to detect } \\
\text { high-risk oral precancerous } \\
\text { lesions in a high-prevalence } \\
\text { and low-resource setting like } \\
\text { India. }\end{array}$ \\
\hline $\begin{array}{l}\text { Rajmohan et al. } \\
2012 \text { (16) }\end{array}$ & $\begin{array}{l}\text { Cross } \\
\text { sectional } \\
\text { study }\end{array}$ & $\begin{array}{l}30 \text { patients (10 } \\
\text { OSCC,9 } \\
\text { dysplasia,1 } \\
\text { benign,10 normal) } \\
\text { Selection criteria: } \\
\text { Group I: Patients } \\
\text { with normal } \\
\text { appearing mucosa } \\
\text { (10) } \\
\text { Group II: } \\
\text { Clinically } \\
\text { diagnosed } \\
\text { precancer (10) } \\
\text { Group III: } \\
\text { clinically } \\
\text { suggestive of } \\
\text { cancer (10) }\end{array}$ & $\begin{array}{l}\text { Assessment of oral } \\
\text { mucosa in normal, } \\
\text { precancer and } \\
\text { cancer using } \\
\text { chemiluminescent } \\
\text { illumination,toulidin } \\
\text { e blue supravital } \\
\text { staining and oral } \\
\text { exfoliative cytology }\end{array}$ & $\begin{array}{l}\text { Dysplasia: } \\
77.8 \% \\
\text { OSCC:90\% }\end{array}$ & Not reported & $\begin{array}{l}\text { OSCC/dysplasia } \\
\text { Negative results in three } \\
\text { erosive lesions . } \\
\text { Conclusion } \\
\text { Vizilite was sensitive for } \\
\text { precancerous and cancerous } \\
\text { lesions with keratotic or red } \\
\text { white characteristics }\end{array}$ \\
\hline
\end{tabular}

OPMD; Oral Potentially Malignant Disorders, OSCC; Oral Squamous Cell Carcinoma.

tive is an erroneously negative test or screening result. Senstivity of Vizilite for detecting OSCC and OPMD ranged from $7.1 \%$ to $100 \%$ and specificity was low that ranged from $0 \%$ to $27.8 \%$. In a study by Ram et al. the sensitivity of vizilite was $100 \%$ and specificity was low $14.2 \%$ (10). Ujaoney et al. found toulidine was better suited than chemiluminescence for detecting high risk patients (15). McIntosh et al. used Microlux DL in his study with sensitivity of $77.8 \%$ and specificity of $70.7 \%$. in detecting dysplasia and OSCC but Microlux TM/DL couldnot discriminate between malignancy, OPMD, benign keratosis and inflammatory conditions (12).
For the use of VELscope in detection of OSCC and OPMD ten studies in literature satisfied our inclusion and exclusion criteria. These studies were mainly crosssectional and were carried out in clinics of countries such as UK (18), Canada (19), Germany (20-22), Italy (23), USA $(24,25)$, Poland (26) and India (14). Clinical trials demonstrated that sensitivity of VELscope in detecting malignancy and OPMD ranged from $22 \%$ to $100 \%$ and specificity ranged from $16 \%$ to $100 \%$.Most studies concluded that VELscope can help the experienced clinician to find oral precursor malignant lesions (20,22,25). Table 2 and 2 continue, summarizes clini- 
Table 2. Summarizes clinical trials to evaluate the efficacy of autofluorescence imaging (VELscope) in detecting oral cancer and oral potentially malignant disorders.

\begin{tabular}{|c|c|c|c|c|c|c|}
\hline $\begin{array}{c}\text { Author } \\
\text { (year) }\end{array}$ & Study design & $\begin{array}{c}\text { Sample, Selection } \\
\text { criteria }\end{array}$ & Intervention & $\begin{array}{l}\text { Outcome: } \\
\text { senstivity }\end{array}$ & $\begin{array}{l}\text { Outcome: } \\
\text { specificity }\end{array}$ & $\begin{array}{c}\text { Summary of findings, } \\
\text { Conclusion }\end{array}$ \\
\hline $\begin{array}{l}\text { Sharwani A et al. } \\
2006 \text { (18) }\end{array}$ & $\begin{array}{l}\text { Cross sectional } \\
\text { study }\end{array}$ & $\begin{array}{l}79 \text { patients } \\
\text { Selection criteria: } \\
\text { Clinically suspicious oral } \\
\text { leukoplakia }\end{array}$ & $\begin{array}{l}\text { Fluorescence } \\
\text { spectroscopy } \\
\text { combined with } \\
\text { 5-aminolevulinic } \\
\text { acid induced } \\
\text { protophyrin IX } \\
\text { fluorescence in } \\
\text { detecting oral } \\
\text { premalignancy }\end{array}$ & $83-90 \%$ & $79-89 \%$ & $\begin{array}{l}\text { Increase in red to green } \\
\text { fluorescence in dysplastic lesion } \\
\text { Conclusion: } \\
\text { Fluorescence spectroscopy } \\
\text { combined with } 5 \text { aminolevulinic } \\
\text { acid induced protophyrin IX } \\
\text { valuable tool in diagnosis of oral } \\
\text { premalignancy }\end{array}$ \\
\hline $\begin{array}{l}\text { Lane et al. } \\
2006(19)\end{array}$ & $\begin{array}{l}\text { Cross sectional } \\
\text { study }\end{array}$ & $\begin{array}{l}44 \text { patients ( } 11 \text { severe } \\
\text { dysplasia,33 OSCC, } 6 \\
\text { normal) } \\
\text { Selection criteria } \\
\text { Oral leukoplakia patients }\end{array}$ & $\begin{array}{l}\text { Direct visualization } \\
\text { of oral cavity tissue } \\
\text { fluorescence }\end{array}$ & $98 \%$ & $100 \%$ & $\begin{array}{l}\text { Conclusion: } \\
\text { Device could be used as an } \\
\text { adjunct to conventional white } \\
\text { light screening to increase the } \\
\text { sensitivity of the white light } \\
\text { screen alone }\end{array}$ \\
\hline $\begin{array}{l}\text { Mehrotra et al. } \\
2010 \text { (14) }\end{array}$ & $\begin{array}{l}\text { Cross sectional } \\
\text { study }\end{array}$ & $\begin{array}{l}156 \text { patients( } 1 \text { OSCC,11 } \\
\text { dysplsia,144 benign } \\
\text { lesions ) } \\
\text { Selection criteria } \\
\text { Oral mucosal white } \\
\text { lesions }\end{array}$ & $\begin{array}{l}\text { Evaluation of } \\
\text { chemiluminescence } \\
\text { and auto fluorescence } \\
\text { in the detection of } \\
\text { clinically innocuous } \\
\text { precancerous and } \\
\text { cancerous oral } \\
\text { lesions }\end{array}$ & $\begin{array}{l}50 \% \\
\text { OSCC:100\% } \\
\text { Dysplasia:45\% }\end{array}$ & $38.9 \%$ & $\begin{array}{l}\text { OSCC/Dysplasia } \\
6 \text { dysplastic lesions did not show } \\
\text { FVL, representing } 6 \text { false negative } \\
\text { screens } \\
\text { Benign lesions } \\
\text { FVL in } 88 \text { benign lesions, } \\
\text { representing false positive screens } \\
\\
\text { Conclusion: } \\
\text { VELscope doesnot add any } \\
\text { benefits to a conventional } \\
\text { screening examination with a } \\
\text { standard overhead light }\end{array}$ \\
\hline $\begin{array}{l}\text { Koch et al. } 2011 \\
\text { (21) }\end{array}$ & $\begin{array}{l}\text { Prospective } \\
\text { blinded clinical } \\
\text { trial }\end{array}$ & $\begin{array}{l}78 \text { patients }(30 \text { OSCC, } 3 \\
\text { dysplasia, } 45 \text { benign) } \\
\text { Selection criteria } \\
\text { OSCC patients or } \\
\text { suspicious epithelial } \\
\text { lesion }\end{array}$ & $\begin{array}{l}\text { Effectiveness of } \\
\text { autofluorescence to } \\
\text { identify suspicious } \\
\text { oral lesions }\end{array}$ & OSCC:93\% & $16 \%$ & $\begin{array}{l}\text { OSCC/Dysplasia } \\
\text { FVL, although highly sensitive } \\
\text {,not very specific for OSCC and } \\
\text { dysplasia } \\
\text { Red color autofluorescence in a } \\
\text { lesion was highly specificto } \\
\text { dysplasia/OSCC (98) but had a } \\
\text { low sensitivity (22\%) } \\
\\
\text { Hyperkeratosis and erythema } \\
\text { VELscope was less able to detect } \\
\text { OSCC with hyperkeraosis.OSCC } \\
\text { with erythema was more likely to } \\
\text { elicit FVL ( } 92 \% \text { sensitive) } \\
\\
\text { Conclusion: } \\
\text { Autofluroscence unable to } \\
\text { differentiate between benign and } \\
\text { malignant lesions.Lesion with red } \\
\text { color autofluorescence should be } \\
\text { biopsed }\end{array}$ \\
\hline $\begin{array}{l}\text { Paderni et al. } 2011 \\
\text { (23) }\end{array}$ & $\begin{array}{l}\text { Cross sectional } \\
\text { study }\end{array}$ & $\begin{array}{l}175 \text { patients }(118 \\
\text { benign,15 mild } \\
\text { dysplasia,14 } \\
\text { moderate/severe } \\
\text { dysplasia,28 OSCC) } \\
\text { Selection criteria: } \\
\text { Patients with atleast one } \\
\text { clinical oral lesion }\end{array}$ & $\begin{array}{l}\text { Direct visualization } \\
\text { of oral cavity tissue } \\
\text { fluorescence as novel } \\
\text { aid for early oral } \\
\text { cancer diagnosis and } \\
\text { potentially malignant } \\
\text { disorders monitioring }\end{array}$ & $\begin{array}{l}\text { OSCC: } 96.4 \% \\
\text { Dysplasia:71\% }\end{array}$ & Not reported & $\begin{array}{l}\text { Conclusion: } \\
\text { Device dosesnot reduce } \\
\text { histopathology procedure }\end{array}$ \\
\hline $\begin{array}{l}\text { Marzouki et al. } \\
2012(24)\end{array}$ & $\begin{array}{l}\text { Prospective single } \\
\text { blind study }\end{array}$ & $\begin{array}{l}85 \text { patients (biopsy } \\
\text { confirmed } 33 \\
\text { OSCC/OPMD) } \\
\text { Selection criteria: } \\
\text { History of smoking, } \\
\text { alcohol use or previous } \\
\text { head and neck cancer }\end{array}$ & $\begin{array}{l}\text { Use of fluorescent } \\
\text { light in detecting } \\
\text { malignant and } \\
\text { premalignant lesions } \\
\text { in oral cavity }\end{array}$ & Dysplasia:92\% & $\begin{array}{l}\text { Dysplasia: } \\
77 \%\end{array}$ & $\begin{array}{l}\text { VELscope showed FVL in } 12 \\
\text { OPMD/dysplasias, } 5 \text { which were } \\
\text { not noted on conventional visual } \\
\text { inspection } \\
\text { VELscope failed to show FVL in } \\
1 \text { OPMD detected on } \\
\text { conventional visual inspection } \\
\text { Conclusion: } \\
\text { VELscope useful tool in high risk } \\
\text { patient }\end{array}$ \\
\hline
\end{tabular}


Table 2 Continue. Summarizes clinical trials to evaluate the efficacy of autofluorescence imaging (VELscope) in detecting oral cancer and oral potentially malignant disorders.

\begin{tabular}{|c|c|c|c|c|c|c|}
\hline $\begin{array}{l}\text { Mc Namara K } \\
\text { et al. } 2012(25)\end{array}$ & $\begin{array}{l}\text { Cross sectional } \\
\text { study }\end{array}$ & $\begin{array}{l}130 \text { patients (1 OSCC, } 2 \\
\text { dysplasia, } 32 \text { benign } \\
\text { lesions) } \\
\text { Selection criteria: } \\
\text { Consecutive recruitment } \\
\text { for routine dental care }\end{array}$ & $\begin{array}{l}\text { Role of VELscope in } \\
\text { routine screening for } \\
\text { potentially malignant } \\
\text { oral mucosal lesions }\end{array}$ & Not reported & Not reported & $\begin{array}{l}\text { OPMD/OSCC } \\
\text { FVL in } 1 \text { malignant and one } \\
\text { dysplastic lesion } \\
\text { Benign } \\
\text { FVL in } 47 \text { either provisionally } \\
\text { diagnosed as benign } \\
\text { Conclusion } \\
\text { VELscope has the potential for } \\
\text { false negatives and has high false } \\
\text { positive rates }\end{array}$ \\
\hline $\begin{array}{l}\text { Rana et al. } 2012 \\
\text { (22) }\end{array}$ & $\begin{array}{l}\text { Cross sectional } \\
\text { study }\end{array}$ & $\begin{array}{l}123 \text { patients (OPMD : } 37 \\
\text { leukoplakia, } 74 \text { lichen } \\
\text { planus, } 2 \text { ulcers, } 2 \\
\text { candida, } 8 \text { others, } 6 \\
\text { dysplasia, } 117 \text { non } \\
\text { dysplastic) } \\
\text { Selection criteria: } \\
\text { Patients with OPMD }\end{array}$ & $\begin{array}{l}\text { Clinical evaluation of } \\
\text { an autofluorescence } \\
\text { diagnostic device for } \\
\text { oral cancer detection }\end{array}$ & $\begin{array}{l}\text { Dysplasia: } \\
100 \%\end{array}$ & $\begin{array}{l}\text { Dysplasia: } \\
74 \%\end{array}$ & $\begin{array}{l}\text { OSCC/Dysplasia } \\
\text { VELscope showed FVL in all } 6 \\
\text { cases of dysplasia } \\
\text { Benign } \\
\text { 37.4\%of all leuko/erythoplakias } \\
\text { and } 81.08 \% \text { of lichen planus } \\
\text { lesions showed FVL. } \\
\text { Conclusion: } \\
\text { VELscope is likely to lead to } \\
\text { overdiagnosis if used by a non } \\
\text { specialist }\end{array}$ \\
\hline $\begin{array}{l}\text { Babiuch et al. } \\
2012 \text { (26) }\end{array}$ & Pilot study & $\begin{array}{l}50 \text { patients } \\
\text { Selection criteria: } \\
\text { Patients with OSCC and } \\
\text { lip cancer }\end{array}$ & $\begin{array}{l}\text { Use of VELscope for } \\
\text { detection of OPMD } \\
\text { and cancers }\end{array}$ & $100 \%$ & $12.5 \%$ & $\begin{array}{l}\text { Autofluorescence was not highly } \\
\text { specific for dysplasias and } \\
\text { cancers, as FVL was observed in } \\
7(87.5 \%) \text { of the benign oral } \\
\text { lesions, leading to a low } \\
\text { specificity of } 12.5 \% \text {.. } \\
\text { Conclusion: } \\
\text { VELscope was useful in } \\
\text { confirming the presence of oral } \\
\text { lesions, the device was unable to } \\
\text { discriminate high - risk from low } \\
\text { - risk lesions }\end{array}$ \\
\hline $\begin{array}{l}\text { Hanken H et al. } \\
2013(20)\end{array}$ & $\begin{array}{l}\text { Single blinded } \\
\text { study }\end{array}$ & $\begin{array}{l}120 \text { patients } \\
\text { Group I examined with } \\
\text { conventional white light } \\
\text { Group II examined with } \\
\text { VELscope } \\
\text { Selection criteria: } \\
\text { Patients with OPMD }\end{array}$ & $\begin{array}{l}\text { Detection of oral } \\
\text { premalignant lesion } \\
\text { with } \\
\text { autofluorescence } \\
\text { based imaging } \\
\text { system-VELscope }\end{array}$ & $22 \%$ & $8.4 \%$ & $\begin{array}{l}\text { Conclusion: } \\
\text { VELscope device is a simple, } \\
\text { non-invasive test of the oral } \\
\text { mucosa, which can help the } \\
\text { experienced clinician to find oral } \\
\text { precursor malignant lesions }\end{array}$ \\
\hline
\end{tabular}

OSCC; Oral squamous cell carcinoma, OPMD; Oral Potentally Malignant Disorders, FVL; Fluorescent visualization loss, VELscope; Visual Enhanced Light scope.

cal trials conducted in literature to test the efficacy of VELscope in early diagnosis of high risk patients and OPMD.

\section{Discussion}

\section{- Chemiluminescence}

Chemiluminescence involves emission of light from a chemical reaction between hydrogen peroxide and acetylsalicylic acid inside a capsule light stick. This reaction emits blue/white light $(430-580 \mathrm{~nm})$ whose principle is based on the reflective properties of tissues that present cellular alterations such as a higher nuclear/cytoplasmatic rate. The acetowhite lesion is more defined, whereas the normal tissue is dark. Chemiluminescence was first applied for the detection of dysplasia in the cervix. The test has recently been adapted and proposed for oral mucosal examination based on the hypothesis that oral mucosal tissues may exhibit features similar to the cervical epithelium when subjected to chemiluminescence (27). One of the components of chemiluminescent examination is acetic acid pre-rinse. It is mainly done to remove the debris and glycoprotein layer for enhanced penetration and reflection of light. But acetic acid is also known to cause cellular dehydration and protein coagulation that reduces the transparency of the epithelium. This could be one of the reasons for the aceto-white appearance of the white lesions (28).

Various studies have been done in literature to evaluate efficacy of Vizilite, some have shown conflicting results. Most have shown that chemiluminescence increases the brightness and margins of oral mucosal white lesions and thus assist in identification of mucosal lesions not detected under Conventional visual examination (COE). Ram et al. found that ViziLite was $100 \%$ 
sensitive with a low specificity of $12.5 \%$ for detection of OPMD and OSCC (10). Rajmohan et al. assessed oral mucosa in normal, precancer and cancer patients using ViziLite and it was found $77.8 \%$ sensitive for detecting dysplasia and $90 \%$ sensitive for detecting OSCC (16). In a study by Awan et al., the majority of mucosal disorders were positive (aceto-white) for chemiluminescence (75.4\%). ViziLite was useful in enhancing the visibility and sharpness of majority of the oral leukoplakia, making the clinically evident lesions more prominent and distinct from surrounding oral mucosa. Fifty percent erythroplakia lesions were ViziLite positive (17).

There are many limitations associated with the use of Vizilite: Examination needs a dark environment, high cost, no permanent record unless photographed,low specificity for dysplasia, contributing to high referral rate and over-treatment, unable to detect some red lesions, acetic acid pre-rinse increases salivary flow that interferes with mucosal surface reflectance, inability to objectively measure the visualization results. This visualization adjunct gives information only about the horizontal extent of the lesion (one dimension). The depth of the lesion which is more important in predicting the malignant behavior cannot be assessed through this modality (11). Various studies proved that Vizilite is not a reliable tool to detect early premalignancy. In a study by Awan et al. majority of leukoplakias $(80.3 \%)$ showed acetowhitening in contrast to only half of the erythroplakias. This clearly demonstrates the ability of the ViziLite to detect leukoplakias (white patches) more accurately and also indicates the inability of ViziLite to detect or enhance some red patches (erythroplakias). The ability of the ViziLite to detect dysplastic lesions has been greatly undermined by failure of the device to distinguish dysplastic from non-dysplastic lesions (sensitivity - 77.3\%, specificity - 27.8\%) (17). Mehrotra et al. found that Vizilite was not sensitive (0\%) in detecting dysplasia and OSCC and has no benefit in detecting OSCC and OPMD (14). Ujaoney et al. used chemiluminescence and Toulidine Blue for detecting of high risk oral precancerous lesions and Toulidine blue was found to be better diagnostic test than chemiluminesecence (15).

- Tissue autofluorescence

The autofluorescence of tissue and its potential use in cancer detection were described first in 1924. It is a phenomenon where by an extrinsic light source is used to excite endogenous fluorophores such as certain amino acids, metabolic products, and structural proteins. Within the oral mucosa, the most relevant fluorophores are nicotinamide adenine dinucleotide (NADH) and flavin adenine dinucleotide (FAD) in the epithelium and collagen cross-links in the stroma. The fluorophores absorb photons from the exogenous light source and emit lower energy photons which present clinically as fluorescence (23). Each fluorophore is associated with specific excitation and emission wavelengths. When irradiated with wavelengths between 375 and $440 \mathrm{~nm}$, the fluorochromes show fluorescence in the green spectral range and normal, unaltered mucosa emits a pale green autofluorescence when viewed through a selective, narrowband filter. A proper filtration is crucial, due to the intense light used for excitation of the fluorochromes. Without a proper filtration, it would be impossible to visualize the pale and narrow autofluorescence signal. However, dysplastic tissues lose fluorescence emission power due to a disruption in the distribution of the fluorochromes and appear darker in colour in comparison to the surrounding healthy tissue (29).

A number of methods based on the principles of tissue fluorescence have been described for use in the oral cavity, including exogenous fluorescence, autofluorescent spectroscopy and autofluorescent imaging. Both exogenous fluorescence and autofluorescent spectroscopy due to practical purposes are unlikely to be applied as screening aids. In exogenous fluorescence, there is a delay before the fluorophore reaches an adequate concentration and the fluorophore also causes temporary photosensitisation to the subject, which may be deemed unacceptable to the individual. In autofluorescence spectroscopy, small optical fibres are used to expose the oral mucosa to different wavelengths of light and it is not possible to screen the entire oral cavity, therefore limiting its application. For these reasons, this review will focus on the use of autofluorescent imaging.

VELscope utilises blue light excitation between 400 and $460 \mathrm{~nm}$ wavelength to enhance oral mucosal abnormalities by direct tissue autofluorescence. At these excitation wavelengths, normal oral mucosa is associated with a pale green fluorescence when viewed through a filter, whereas abnormal tissue is associated with a loss of autofluorescence and appears dark. Neoplastic tissues are expected to cause fluorescent visualisation loss (FVL) and thus appear as a dark area (30).

Several studies have investigated the effectiveness of the VELscope system as an adjunct to visual examination for 1) improving the distinction between normal and abnormal tissues (both benign and malignant changes) 2) differentiating between benign and dysplastic/malignant changes 3 ) and identifying dysplastic/malignant lesions that are visible to naked eye under white light. Whether it can distinguish between dysplasia and benign inflammatory lesions is questioned. Benign inflammatory conditions can result in an increased blood supply to a lesion. The increased haemoglobin content (chromophores) may absorb light and cause FVL mimicking neoplasia $(24,25)$.

Hanken $\mathrm{H}$ et al. examined 120 patients with suspicious oral lesions and found VELscope has a higher sensitivity $(22.0 \%)$, and a lower specificity $(8.4 \%)$. Also it is more promising than $\mathrm{COE}$ in detecting precursor oral 
malignant lesions (20). Koch et al. in his study showed a higher sensitivity $(97 \%)$ and specificity of $(95.8 \%)$ of VELscope to diagnose OSCC. The positive predictive value (PPV) was calculated was $41 \%$ and negative predictive value (NPV) was $75-80 \%$ (21). Rana et al. in his study showed that using the VELscope leads to higher sensitivity (100\% vs. $17 \%)$, but a lower specificity ( $74 \%$ vs. $97 \%$ ) as compared to COE. The major lack of the study was the large number of false-positive test results (22). In another study McNamara et al. concluded that COE is more valid than autofluorescence examination with VELscope in routine screening for OPMD (25). They believed that careful, systematic visual and tactile examination of the entire oral cavity on a regular basis remains the gold standard for early detection of OPMD. Babiuch et al. found in his study that autofluorescence was not highly specific for dysplasias and cancers, as FVL was observed in $7(87.5 \%)$ of the benign oral lesions, leading to low specificity of $12.5 \%$. But this device was unable to discriminate high risk from low risk lesions (26).

\section{Conclusions}

Detection of OPMDs before they advance to OSCC is necessary to improve survival rates for oral cancer. Evidence indicates that $\mathrm{COE}$ is a poor discriminator of oral mucosal lesions, and this has led to the development of several adjunctive visualisation aids. Both devices are simple, non-invasive tests of the oral mucosa, which can help the experienced clinician to find oral precursor malignant lesions and the correct location for taking biopsies within the altered mucosa. But in the literature, both techniques have limited ability to discriminate the high-risk lesions and have limitations which limit their use. In any case, conventional visual inspection under normal incandescent light, followed by biopsy of suspicious lesions, will remain the gold standard for the immediate future. Future approaches to optical imaging could involve real time quantitative evaluation to determine a diagnosis for oral mucosal lesions rather than simply highlighting the presence of abnormalities, thus, making the possibility of "optical biopsy" a clinical reality.

\section{References}

1. Marocchio LS, Lima J, Sperandio FF, Corrêa L, de Sousa SO. Oral squamous cell carcinoma: an analysis of 1,564 cases showing advances in early detection. J Oral Sci. 2010;52:267-73.

2. Gaikwad P, Hiremath SKS, Singh S. Advancement in Diagnostic aids for oral premalignant lesions:A review. J Dent Sci Oral Rehabil. 2013;11:5.

3. Sciubba JJ. Oral cancer. The importance of early diagnosis and treatment. Am J Clin Dermatol. 2001;2:239-51.

4. Warnakulasuriya S. Causes of oral cancer--an appraisal of controversies. Br Dent J. 2009;207:471-5.
5. Brocklehurst P, Kujan O, Glenny AM, Oliver R, Sloan P, Ogden G, et al. Screening programmes for the early detection and prevention of oral cancer. Cochrane Database Syst Rev. 2010;11:CD004150.

6. Garg P, Karjodkar F. Catch Them before it Becomes Too LateOral Cancer Detection. Report of Two Cases and Review of Diagnostic AIDS in Cancer Detection. Int J Prev Med. 2012;3:737-41.

7. Gillenwater A, Jacob R, Richards-Kortum R. Fluorescence spectroscopy: a technique with potential to improve the early detection of aerodigestive tract neoplasia. Head Neck. 1998;20:556-62.

8. Downer MC, Moles DR, Palmer S, Speight PM. A systematic review of test performance in screening for oral cancer and precancer. Oral Oncol. 2004;40:264-73.

9. Scully C, Bagan JV, Hopper C, Epstein JB. Oral cancer: current and future diagnostic techniques. Am J Dent. 2008;21:199-209.

10. Ram S, Siar CH. Chemiluminescence as a diagnostic aid in the detection of oral cancer and potentially malignant epithelial lesions. Int J Oral Maxillofac Surg. 2005;34:521-7.

11. Farah CS, McCullough MJ. A pilot case control study on the efficacy of acetic acid wash and chemiluminescent illumination (ViziLite Trade mark) in the visualisation of oral mucosal white lesions. Oral Oncol. 2007;43:820-4.

12. McIntosh L, McCullough MJ, Farah CS. The assessment of diffused light illumination and acetic acid rinse (Microlux/DLTM) in the visualisation of oral mucosal lesions. Oral Oncol. 2009;45:e22731.

13. Epstein JB, Silverman S Jr, Epstein JD, Lonky SA, Bride MA. Analysis of oral lesion biopsies identified and evaluated by visual examination, chemiluminescence and toluidine blue. Oral Oncol. 2008; $44: 538-44$

14. Mehrotra R, Singh M, Thomas S, Nair P, Pandya S, Nigam NS, et al. A cross-sectional study evaluating chemiluminescence and autofluorescence in the detection of clinically innocuous precancerous and cancerous oral lesions. J Am Dent Assoc. 2010;141:151-6.

15. Ujaoney S, Motwani MB, Degwekar S, Wadhwan V, Zade P, Chaudhary M, et al. Evaluation of chemiluminescence, toluidine blue and histopathology for detection of high risk oral precancerous lesions: A cross-sectional study. BMC Clin Pathol. 2012;12:6.

16. Rajmohan M, Rao UK, Joshua E, Rajasekaran ST, Kannan R. Assessment of oral mucosa in normal, precancer and cancer using chemiluminescent illumination, toluidine blue supravital staining and oral exfoliative cytology. J Oral Maxillofac Pathol. 2012;16:325-9.

17. Awan KH, Morgan PR, Warnakulauriya S. Utility of chemiluminescence (ViziLiteTM) in the detection of oral potentially malignant disorders and benign keratoses. J Oral Pathol Med. 2011;40:541-4. 18. Sharwani A, Jerjes W, Salih V, MacRobert AJ, El-Maaytah M, Khalil HS, et al. Fluorescence spectroscopy combined with 5-aminolevulinic acid-induced protoporphyrin IX fluorescence in detecting oral premalignancy. J Photochem Photobiol B. 2006;83:27-33. 19. Lane PM, Gilhuly T, Whitehead P, Zeng H, Poh CF, Ng S, et al. Simple device for the direct visualization of oral-cavity tissue fluorescence. J Biomed Opt. 2006;11:024006.

20. Hanken H, Kraatz J, Smeets R, Heiland M, Assaf At, Blessmann $\mathrm{M}$, et al. The detection of oral pre malignant lesions with an autofluorescence based imaging system (VELscope TM) - A single blinded clinical evaluation. Head Face Med. 2013;9:23.

21. Koch FP, Kaemmerer PW, Biestergeld S, Kunkel M, Wagner W. Effectiveness of autofluorescence to identify suspicious oral lesionsa prospective, blinded clinical trial. Clin Oral Investig. 2011;15:97582.

22. Rana M, Zapf A, Kuehle M, Gellrich NS, Eckardt AM. Clinical evaluation of an autofluorescence diagnostic device for oral cancer detection: a prospective randomized diagnostic study. Eur J Cancer Prev. 2012;21:460-6.

23. Paderni C, Compilato D, Carinci F, Nardi G, Rodolico V, Lo Muzio L, et al. Direct visualization of oral-cavity tissue fluorescence as novel aid for early oral cancer diagnosis and potentially malignant disorders monitoring. Int J Immunopathol Pharmacol. 2011;24(2 Suppl):121-8. 
24. Marzouki HZ, Tuong VVT, Ywakin R, Chauvin P, Hanley J, Kost KM. Use of fluorescent light in detecting malignant and premalignant lesions in the oral cavity: a prospective, single-blind study. J Otolaryngol Head Neck Surg. 2012;41:164-8.

25. McNamara KK, Martin BD, Evans EW, Kalmar JR. The role of direct visual fluorescent examination (VELscope) in routine screening for potentially malignant oral mucosal lesions. Oral Surg Oral Med Oral Pathol Oral Radiol. 2012;114:636-43.

26. Babiuch K, Chomyszyn-Gajewska M, Wyszyńska-Pawelec G. Use of VELscope for detection of oral potentially malignant disorders. and cancers. Medical and Biological Sciences. 2012;26:11-6.

27. Oh ES, Laskin DS. Efficacy of vizilite system in identification of oral lesions. J Oral Maxill Surg. 2007;65:24-7.

28. Shedd DP, Hukill PB, Bahn S, Farraro RH. Further appraisal of in vivo staining properties of oral cancer. Arch Surg. 1967;95:16-22.

29. Betz CS, Stepp H, Janda P, Arbogast S, Grevers G, Baumgartner $\mathrm{R}$, et al. A comparative study of normal inspection, autofluorescence and 5-ALA-induced PPIX fluorescence for oral cancer diagnosis. Int J Cancer. 2002;97:245-52.

30. Poh CF, MacAulay CE, Zhang L, Rosin MP. Tracing the "at-risk" oral mucosa field with autofluorescence: steps toward clinical impact. Cancer Prev Res (Phila). 2009;2:401-4.

Conflict of Interest

The authors of this paper have no conflict of interest to report regarding this publication. 